\title{
Atypical Noncontiguous Multiple Spinal Tuberculosis: A Case Report
}

\author{
Jang-Hoon Kim, Se-Hoon Kim, Jong-II Choi, Dong-Jun Lim \\ Department of Neurosurgery, Ansan Hospital, Korea University College of Medicine, Seoul, Korea
}

\begin{abstract}
Objective: Spinal tuberculosis-associated symptoms are not so unique as to immediately indicate the proper diagnosis in most cases. Distinguishing spinal tuberculosis (Pott's disease) from pyogenic spondylitis is often difficult, and lesions metastatic from systemic malignancy are the other major entity from which spinal tuberculosis must be distinguished.

Clinical Presentation: A 27-year-old male patient presented with a history of back pain after a minor trauma 1 month ago. Computed tomography and magnetic resonance imaging of the thoracic spine showed multiple osteolytic bone lesions at the bodies of T9, T10 and T11 vertebrae and the spinous processes of T12 and L1. Other noncontiguous osteolytic lesions were noted at S2 body and right sacro-liac joint.

Intervention: To confirm the pathologic diagnosis, the patient underwent an open biopsy for the T12 and L1 spinous process lesions and a percutaneous transpedicular biopsy on T9, T10, T11 lesions. Frozen biopsy was reported as compatible with chronic granulomatous caseating necrosis without malignant cells. The final diagnosis was an atypical presentation of multiple spinal tuberculosis. The patient received an appropriate enteral anti-tuberculosis therapy and recovered without any complications. Follow-up MRI taken after a year of medical treatment revealed marked resolution of the lesions.

Conclusion: Current research indicates the incidence of multi-level noncontiguous, remote vertebral tuberculosis is $1.1 \%$ to $16 \%$. Because tuberculous spondylitis could represent variant and atypical pattern, the disease should be considered in differential diagnosis along with other diseases such as metastatic neoplasm, pyogenic spondylitis, especially when the radiologic studies are revealing multiple spinal lesions.
\end{abstract}

Key Words: Spondylitis · Spinal tuberculosis $\cdot$ Pott's disease

\section{INTRODUCTION}

Features of spinal tuberculosis (Pott's disease), an extrapulmonary form of tuberculosis, include destruction of the intervertebral space and collapse of vertebral bodies, leading to progressive kyphosis. The thoracic spine is the most common site of involvement ${ }^{1}$. Spinal tuberculosis used to be an uncommon disease in developed countries, and its associated symptoms are not so unique as to immediately indicate the proper diagnosis in most of cases $^{2}$. Distinguishing spinal tuberculosis

\footnotetext{
- Received: February 20, 2014 - Revised: March 4, 2014

- Accepted: March 4, 2014

Corresponding Author: Se-Hoon Kim, MD, PhD

Department of Neurosurgery, Ansan Hospital, Korea University Medical Center, 123 Jeokgeum-ro, Danwon-gu, Ansan, Gyeonggi-do 425-707, Korea Tel: +82-31-412-5050, Fax: +82-31-412-5054

E-mail: sean1050@gmail.com

*Disclosure: The authors have no personal financial or institutional interest in any of the drugs, materials, or devices described in this article. No financial support received in conjunction with the generation of this submission.

œThis is an Open Access article distributed under the terms of the Creative

Commons Attribution Non-Commercial License (http://creativecommons.org/ licenses/by-nc/3.0/) which permits unrestricted non-commercial use, distribution, and reproduction in any medium, provided the original work is properly cited.
}

from pyogenic osteomyelitis, however, is often very difficult and, lesions metastatic from systemic malignancy are the other major entity from which spinal tuberculosis must be distinguished $^{2}$.

Usually, two or more contiguous vertebrae are involved in spinal tuberculosis due to hematogenous spread through one vertebral artery feeding two adjacent vertebrae ${ }^{3,4)}$. Noncontiguous, multiple, remote involvement of tuberculous spondylitis is relatively rare, and most of the reported cases have lesions only in two levels ${ }^{3-6}$. Cur rent research indicates the incidence of multiple-level noncontiguous vertebral tuberculosis is 1.1\% to $16 \% \%^{7}$. We present a case of atypical spinal tuberculosis in a 27-year-old man with noncontiguous multiple spinal lesions.

\section{CASE REPORT}

A 27-year-old male patient presented with a 1-month history of pain in his back after a minor trauma 1 month ago. He had no history of systemic disease. There was no visible or palpable deformity but tenderness could be elicited over the spinous processes of the lower thoracic vertebrae. Neurologic examination represented no specific findings. Plain radio- 


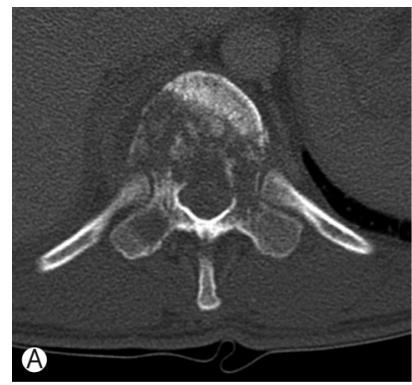

Fig. 1. Initial oxial computed tomography at the level of $\mathrm{T} 11$ (A) and $3 \mathrm{D}$ reconstruction $(\mathrm{B})$ images of the thoracic vertebrae reveal multiple destructive lesions of the T9, T10 and T11 vertebral bodies and spinous process of $\mathrm{T} 12$.
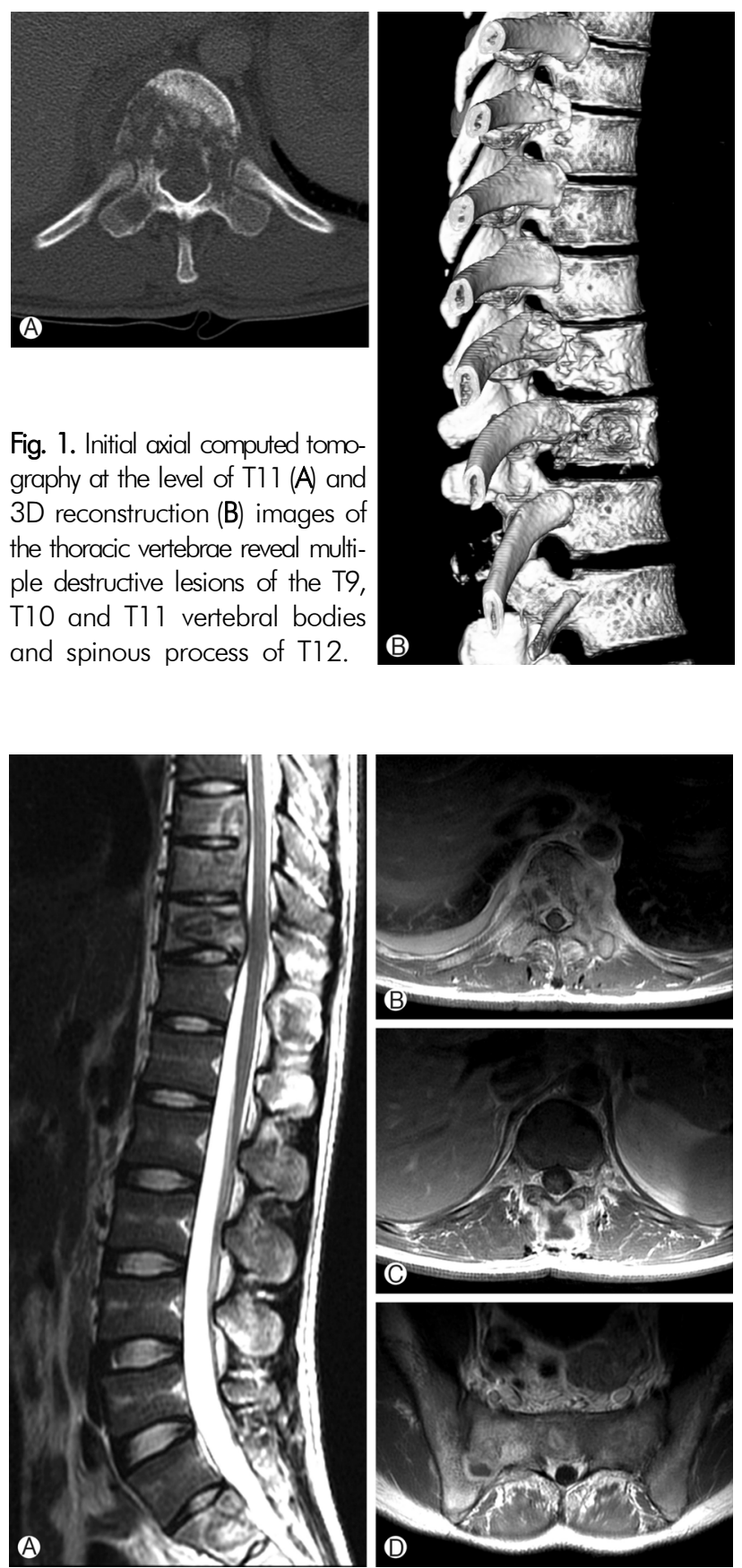

Fig. 2. Initial thoracolumbar spine MRI. Sagittal T2-weighted image (A) shows heterogeneous, mostly hyperintense lesions involving the T9, T10, T1 1 and S2 vertebral bodies and the spinous processes of T12 and L1. Gadolinium-enhanced axial T1-weighted images demonstrate heterogeneous enhancement of the T11 vertebral body (B) and T12 spinous process (C) as well as the S2 body and the right sacro-iliac joint (D). The disc spaces are relatively preserved.

graphs demonstrated multiple destructive changes in the thoracic spines. Computed tomography (CT) and magnetic resonance imaging (MRI) of the thoracic spine showed multiple

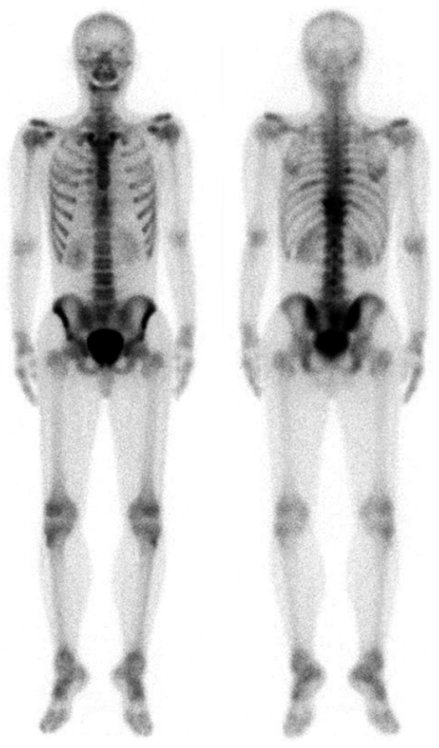

Fig. 3. Whole body $99 \mathrm{mTc}-$ HMDP bone scan shows multiple increased uptakes in the lower thoracic vertebrae, sacrum and the right sacro-iliac joint.

osteolytic bone lesions. At the bodies of T9, T10 and T11 vertebrae, osteolytic bone lesions were noted along with the similar lesions at the spinous processes of T12 and L1. Other noncontiguous osteolytic lesions were also noted at S2 body and right sacro-iliac joint on the further MRI study. On enhanced studies, these lesions were well enhanced with heterogeneous pattern (Fig. 1, 2). Chest X-ray and enhanced chest CT studies did not show any parenchymal lung lesion or lympho-adenopathy, and three times of sputum acid fast bacilli stain showed all negative results. Complete blood count, routine biochemistries, urinalysis, liver function tests, thyroid function tests, lipid profiles, and creatine kinase level were all normal. The erythrocyte sedimentation rate and C-reactive protein was normal. Anti-human immune deficiency virus (HIV) antibodies, hepatitis B surface (HBS) antigen, and hepatitis C virus (HCV) antigen were all negative. Venereal disease research laboratory (VDRL), syphilitic serology tests, and serum tumor markers were all within normal ranges. Whole body bone scan showed multiple hot uptakes in the lower thoracic spines, sacrum and sacro-iliac joint on the right (Fig. 3).

To confirm the pathologic tissue diagnosis, the patient underwent an open biopsy for the T12 and L1 spinous process lesions through a midline small skin incision and a percutaneous transpedicular biopsy on T9, T10, T11 lesions under general anesthesia. We found a cheese-like material in the T12 spinous process. Frozen biopsy was reported as compatible with chronic granulomatous caseating necrosis without malignant cells. The specimens were further checked by polymerase chain reaction (PCR) test for tuberculosis and reported as positive. The final diagnosis was an atypical presentation of multiple spinal tuberculosis. The patient received an appropriate enteral anti-tuberculous therapy and recovered without 


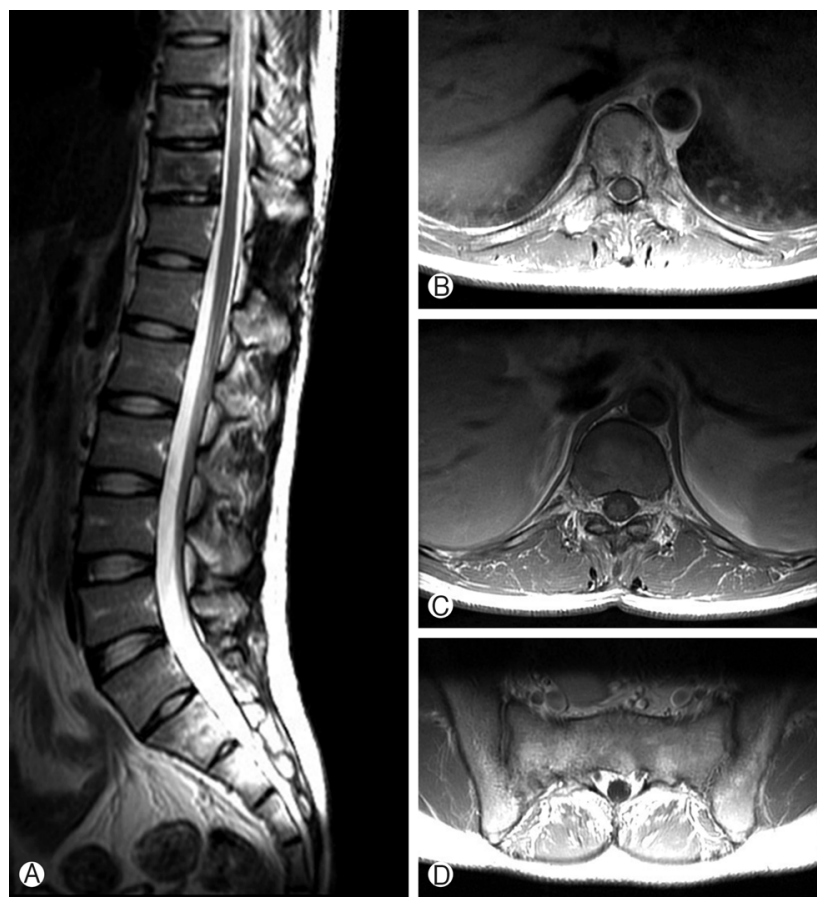

Fig. 4. Follow-up MRI of the thoracolumbar spine taken a year after the anti-tuberculous therapy. Sagittal T2-weighted image (A) and Gadolinium-enhanced axial T1-weighted images at the levels of $\mathrm{T} 11, \mathrm{~T} 12$ and S2 (B, C, D) demonstrate marked resolution of the lesions.

any complications. Follow-up MRI taken after a year of anti-tuberculous therapy revealed marked resolution of the lesions (Fig. 4).

\section{DISCUSSION}

Tuberculosis, a disease that once was on the decline and almost eradicated in the first world, has made a dramatic comeback. According to the World Health Organization, tuberculosis is the number one specific infectious cause of death worldwide $^{8}$. It is estimated that one-third of the world's population is infected with tuberculosis, an estimated 2 billion people. South Africa has the world's highest reported incidence of tuberculosis at 718/100,000 reaching $1.2 \%$ of the population in certain areas ${ }^{8)}$.

Extrapulmonary tuberculosis consists of $15-20 \%$ of the patients if you include pleural and lymphatic disease, which is most common ${ }^{8)}$. Skeletal tuberculosis is $10 \%$ of this, of which spinal tuberculosis accounts for approximately 50\%. This gives an incidence of between 1 and 2\% for osteoarticular tuberculosis and $0.5-1 \%$ for spinal tuberculosis ${ }^{9}$. Tuberculosis has a worthy reputation as one of the great mimickers in medicine with a multitude of clinical pictures and variations. This makes the characteristic features of the classic disease problematic, with most texts and articles describing a typical clinical and radiological picture followed by many atypical derivatives. Although tuberculous spondylitis frequently involves multiple adjacent spinal vertebrae, noncontiguous, remote involvement is reported as rare in literature ${ }^{3-6,8)}$.

A feature most typically distinguishing spinal tuberculosis from pyogenic osteomyelitis is the relative sparing of the intervertebral discs. Mycobacteria lack the proteolytic enzymes found in bacteria that commonly cause pyogenic osteomyelitis, and this may be responsible for the relative sparing of the disc ${ }^{10}$. The avascularity of the discs may prevent them from serving as an initial site of infection, and it has been suggested that disc destruction begins only when the two adjacent vertebral bodies are so involved that the disc loses its nutritional support. Pott's disease can be also distinguished by its characteristic paravertebral abscesses. The extent of these collections may greatly exceed the area of osseous involvement. These abscesses may be located anterior, posterior, lateral, or circumferentially around the vertebral body. The wall of the abscess is characteristically thick and irregularly enhancing on both CT and $\mathrm{MR}$ images, and this feature is thought to be diagnostic of tuberculous spondylitis ${ }^{2)}$.

Multiple metastatic spinal lesions from systemic malignancy are the major entity from which multiple spinal tuberculosis must be distinguished. Metastases characteristically spare the disc space, as does spinal tuberculosis. When the spinal tuberculosis involves multiple noncontiguous vertebrae, its imaging appearance can be easily mistaken for metastatic malignancy. Factors that should indicate and distinguish spinal tuberculosis from neoplastic disease include the presence of paravertebral abscesses and subligamentous spread ${ }^{2}$. In our case, tuberculosis involved mainly he vertebral bodies not the pedicles, that impressed spinal tuberculosis rather than other malignancy.

In the presenting case of the authors, the pattern of vertebral infiltration was very atypical and variable along the spinal levels. The osteolytic pattern of T9, T10, T11 vertebral bodies could be considered as relatively typical form of spinal tuberculosis. There was, however, no evidence of bony involvement or paravertebral abscess formation in the anterior or middle columns of T12 and L1 vertebrae, and spinous processes were only involved. Especially at the level of T12, spinous process was destroyed near totally but pedicles and vertebral body was intact in the imaging studies. Another atypical pattern of our case was its discontinuity. Thoracic and lumbar vertebrae, sacrum, and iliac bone were involved simultaneously with discontinuity. Noncontiguous spinal tuberculosis is far more common (16.3\%) than that reported in literature ${ }^{8)}$. There was a trend towards a higher incidence of neurology in the noncontiguous group compared to the single focus ${ }^{8}$. 
This trend may not necessarily reflect more severe disease, but instead may be a product of heightened probability due to the increased levels involved and/or the double-crush neural phenomenon. Noncontiguous spinal tuberculosis is not an obvious manifestation of $\mathrm{HIV}$, multi-drug resistant (MDR) tuberculosis or chronicity as in our patient. It is not a "fulminant" type of tuberculosis as suggested by Emel, et al ${ }^{33}$.

Some articles reported the role of bone scan in the spinal tuberculosis. Evangelista, et al reported that bone scan can anticipate radiograph by 2 to 5 months and help guidance of the site of biopsy.11 Although skeletal locations mostly involve the dorsolumbar spine, nuclear imaging allows assessment of the whole body. In our case, MRI and whole body bone scan revealed remote noncontiguous lesions, though patient did not complained of any symptoms on sacrum or sacro-iliac area.

Atypical osseous tuberculosis lesions of the spine share several features in common as reported by Naim-Ur-Rahman, et $\mathrm{al}^{11,12}$. First, atypical vertebral lesions readily involve the neural arch, sparing the vertebral body and discs (referred to as "posterior Pott"). Second, skip lesions are often present, as defined by the presence of two or more vertebral lesions separated by an uninvolved vertebra. Finally, lesions may manifest as solid tumor-like masses with no evidence of abscess formation, discovered at the time of surgery and indistinguishable from a solid spinal extradural tumor. In the present case, imaging study revealed such a similar pattern of osseous lesions, and involvement of the neural arch of T12 and L1 was first observed on the MRI. Skip lesions were also observed in our patient. Asymptomatic lesions of sacrum and the right sacro-iliac joint were identified as a scintigraphic pattern of multiple, disseminated foci of uptake together with normal uptake of other vertebrae. Finally, scintigraphic heterogeneity of the right sacroiliac lesion could be interpreted as a tumor-like lesion. When symptomatic, tuberculous sacro-iliitis can present with nonspecific complaints of buttock pain, which may also evoke several pathologies, such as psoas abscess, appendicitis, sciatica or pyelonephritis, and some primary bone tumors like chordoma or chondrosarcoma ${ }^{12}$.

As our case with neurologically intact condition or with minimal systemic symptoms, most patients demonstrate good outcomes with standard care ${ }^{8}$.

\section{CONCLUSION}

Tuberculosis is not a rare disease even in the developed countries these days. Metastatic neoplasm, tuberculous spondylitis, and pyogenic spondylitis must be considered in the differential diagnosis of multiple spinal lesions. Because spinal tuberculosis could represent a variant and atypical pattern, it should be kept in mind that appropriate radiologic examinations, such as whole body bone scan or spine MRI, are useful in osseous tuberculosis, especially when the lesions are multiple.

\section{REFERENCES}

1. Sarangapani A, Fallah A, Provias J, Jha NK.: Atypical presentation of spinal tuberculosis. Can J Surg 51:E121, 2008

2. Joseffer SS, Cooper PR.: Modern imaging of spinal tuberculosis. J Neurosurg Spine 2:145-150, 2005

3. Emel E, Guzey FK, Guzey D, Bas NS, Sel B, Alatas I: Non-contiguous multifocal spinal tuberculosis involving cervical, thoracic, lumbar and sacral segments: a case report. Eur Spine J 15: 1019-1024, 2006

4. Kulali A, Cobanoğlu S, Ozyilmaz F. Spinal tuberculosis with circumferential involvement of two noncontiguous isolated vertebral levels: case report. Neurosurgery 35:1154-1158, 1994

5. Janssens JP, de Haller R. Spinal tuberculosis in a developed country: A review of 26 cases with special emphasis on abscesses and neurologic complications. Clin Orthop Relat Res 257:6775,1990

6. Turgut M: Spinal tuberculosis (Pott's disease): its clinical presentation, surgical management, and outcome. A survey study on 694 patients. Neurosurg Rev 24:8-13, 2001

7. Kaila R, Malhi AM, Mahmood B, Saifuddin A.: The incidence of multiple level noncontiguous vertebral tuberculosis detected using whole spine MRI. J Spinal Disord Tech 20:78-81, 2007

8. Polley P, Dunn R.: Noncontiguous spinal tuberculosis: incidence and management. Eur Spine J 18:1096-1101, 2009

9. Naim-Ur-Rahman: Atypical forms of spinal tuberculosis. J Bone Joint Surg Br 62:162-165, 1980

10. Chapman M, Murray RO, Stoker DJ.: Tuberculosis of the bones and joints. Semin Roentgenol 14:266-282, 1979

11. Evangelista E, Itti E, Malek Z, et al.: Diagnostic value of $99 \mathrm{mTc}-$ HMDP bone scan in atypical osseous tuberculosis mimicking multiple secondary metastases. Spine (Phila Pa 1976) 29:E8587, 2004

12. Naim-Ur-Rahman, El-Bakry A, Jamjoom A, Jamjoom ZA, Kolawole TM.: Atypical forms of spinal tuberculosis: case report and review of the literature. Surg Neurol 51:602-607, 1995 